\title{
Association of Physical Activity, Screen Time and Sleep with Depressive Symptoms in Adolescents
}

\author{
Qiang Sun ${ }^{1}$ and Xuzhi Zhan ${ }^{2, *}$ \\ ${ }^{1}$ Center of Jiangsu Sports Health Engineering Collaborative Innovation, Nanjing Sport Institute, Nanjing, 210014, China \\ ${ }^{2}$ School of Physical Education and Humanity, Nanjing Sport Institute, Nanjing, 210014, China \\ *Corresponding Author: Xuzhi Zhan. Email: zhanxuzhi2020@163.com
}

Received: 14 October 2020 Accepted: 21 December 2020

\begin{abstract}
Little is known on the association between movement behaviors including physical activity (PA), screen time (ST) and sleep (SLP) with depression in adolescents. This study aimed to explore the associations of PA, ST and SLP with depressive symptoms in adolescents. A total of 1,331 middle school students participated in this survey and provided valid data pertaining to the study variables. Self-reported questionnaires were used to collect information on participants' sociodemographic parameters. The Health Behavior in School-aged Children Questionnaire was used to assess the PA (days for moderate to vigorous PA), ST (daily hours of ST) and SLP (daily hours of SLP). Study participants' depressive symptoms were assessed using the Children's Depression Inventory. Generalized linear models were used to estimate the associations of PA, ST and SLP with depressive symptoms. Of all the 1331 study participants, boys accounted for $51.31 \%$ and percentage of $7^{\text {th }}, 8^{\text {th }}$ and $9^{\text {th }}$ graders were $52.44 \%$, $23.22 \%$ and $24.34 \%$, respectively. After controlling for sex, body mass index, grade, ethnicity, residence, siblings, perceived family affluence, father and mother educational level, only ST was positively associated with depressive symptoms (beta $=0.17, p=0.005)$. This study suggests that excessive ST would be detrimental to depressive symptoms in adolescents. It may be effective to reduce ST for depression intervention or prevention. Future studies are encouraged to use an improved study design to confirm or negate this study's research findings.
\end{abstract}

\section{KEYWORDS}

Moderate to vigorous physical activity; screen time; sleep; depression; middle school students

\section{Introduction}

Depression is a leading factor of death by suicide in adolescents [1] and is an essential indicator of mental disorders and social problems in adults [2,3]. The prevalence of depressive disorder among adolescents has been estimated to be much higher in the year of 2030 [4,5], with the growing prevalence of symptoms being observed in increasing recently published studies [6]. Many lifestyle risk factors (e.g., physical activity [PA], screen time [ST] or sleep) could lead to a climbing prevalence of depressive symptoms, and identifying modifiable behaviors associated with them is imperative for informing effective and preventive strategies and interventions for those population who are at risk, where adolescents are more vulnerable to be depressive [7]. 
Since the popularity of time-use epidemiology framework, an increasing number of studies have started combining PA, ST and SLP together in health filed research. The framework stresses that PA, ST and SLP are co-dependent behaviors. Based on this framework, it is necessary to included PA, ST and SLP together when studying some relevant studies. There have been sufficient interests in exploring the roles of movement behavior comprising PA, ST and SLP on depressive symptoms in adolescents in the literature. Movement behaviors explain the day of each individual because they are mutually exclusive from a 24hour perspective; that is, $1 \mathrm{~min}$ spent in one of these behaviors implies $1 \mathrm{~min}$ less spent in the others [8]. The single relationship (e.g., PA) of one behavior with depressive symptoms has been investigated in many studies previously. For instance, PA has been highlighted by a review including prospective studies, which indicated that PA was a protective factor against depressive symptoms [9], but associations were not conclusive in studies of adolescents; the negative associations between PA and depressive symptoms in adolescents were found as well [10-12]. ST has been shown as a correlate of depressive symptoms in adolescents [10,13] and sleep also seems to be inversely associated with depressive symptoms in adolescents [10,14-16]. Overall, the available evidence suggests that more PA, reduced SB, and appropriate sleep are independent protective factors for depressive symptoms. However, owing to the independent association of PA, SB and SLP with depression symptoms in adolescents, it is highly unreliable to assess the association between single one behavior (e.g., PA, SB or SLP) with depression symptoms, respectively, while not controlling the others [17]. On this regard, an increasing number of studies have started to examine the association between concurrence of PA, SB and SLP with depression symptoms [18,19].

However, those studies were mostly based on western adolescents instead of Chinese populations, which may inhibit our understanding of depression symptoms prevention from the perspective of movement behavior. In addition to the consideration, few studies is consisting of PA, ST and SLP concurrently to assess the association with depression symptoms. It is therefore required to add more evidence in this research field. Thus, this study aimed to explore the association of PA, ST and SLP with depression symptoms in adolescents of a Chinese sample. Based on previous studies, the research hypothesis in the current study is that PA, ST and SLP will be significantly correlated with depressive symptoms in adolescents.

\section{Methods}

\subsection{Study Design and Participants}

Our study was designed to assess the factors on adolescents' physical and mental health during summertime. We conducted a cross-sectional study to achieve our study aims and adolescents (middle school students of grade 7-9) were recruited into this study. We randomly selected one middle school (having three grades) in each city (13 cities in our study) of Jiangsu Province. In each grade, students of three class were selected randomly. In total, 3465 study participants were invited into this study. 1998 study participants and their parents completed the survey and the response rate of this study was not high (response rate $=57.7 \%$ ). Of the 1998 study participants, 1331 (missing cases of sex $=125$; missing cases of grade $=95$; missing cases of height and weight $=88$; missing cases of father and mother education level $=134$; missing cases of $\mathrm{PA}=105$, missing cases of ST $=56$; missing cases of SLP $=64$ ) study participants provided valid data on the variables of this study. Before data collection, the headmaster of each school, study participants and their parents were informed study aims and instructions on the survey. Each study participants and their parents provide written consent to take part in this study. To protect study participants' privacy, data were collected and analyzed anonymously.

\subsection{Procedure and Data Collection}

The survey was conducted online for both convenience and safety reasons in light of the COVID-19 outbreak. Over two weeks, students were invited to participate in the survey via Tencent's QQ, WeChat, Weibo. Recruitment and data collection procedures were approved by the Human Research Ethics Committee of Nanjing Sport Institute (Nanjing, China). 


\subsection{Measures}

\subsubsection{Sociodemographic}

Study participants were asked to self-reported the following information, including sex (boy or girl), birthdate (year/month), Grade $(7,8,9)$, ethnicity (Han or minority), residence (urban or rural), height $(\mathrm{cm})$ and weight $(\mathrm{kg})$, perceived family affluence (scores from; using Family Affluence Scale), father and mother's educational level (below the primary school, primary school, middle school, high or occupation school, three-college undergraduate, postgraduate or above), siblings (none, one, two, three, four or more).

\subsubsection{Study Outcome (Depression Symptoms)}

The study outcome was depression symptoms. Study participants' depression symptoms were assessed by a self-reported measure, that is Children's Depression Inventory (CDI). The measure was developed by Kovacs et al. and has been validated in Chinese school-aged children and adolescents aged 7-17 years (reliability coefficient: Cronbach's $\alpha$ coefficient $=0.88$ and test-retest reliability coefficient $=0.93$; validity coefficient: 0.43 ). The CDI consisted of 27 items and their responses ranged from 0 to 2 , generating a total of $0-54$ scores. The higher scores participants reported, the depressive severity participants rated.

\subsubsection{Study Exposure (Movement Behaviors)}

MVPA was assessed by the Health Behavior in School-aged Children (HBSC) [20] Questionnaire that has been used for many Chinese epidemiological surveys [21-24]. One validated question (test-retest reliability coefficient $=0.82$ ) that is how many days for engagement in physical activity that increases your heartbeat and make you breathe hard some of the time (including physical education time, exercise, sports training, and various regular daily activities, such as brisk walking, hiking, and excursion) (e.g., physical education, exercise, sport participations). The responses were 0-7 days. In the statistical analysis, the variable of MVPA was treated as continuous.

Screen time was assessed by the HBSC questionnaire [20], the questions included: (1) How many hours did you spend watching TV or movies in your leisure time on weekdays and weekend days over the past week, respectively? (2) How many hours did you spend playing video games in your leisure time on weekdays and weekend days over the past week, respectively? (3) How many hours did you spend in activities using electronic screen-based devices in leisure time on weekdays and weekend days over the past week, respectively? The test retest reliability coefficient ranged from 0.33 to 0.74 . The responses to these questions were $0.5 \mathrm{~h}$ to $7 \mathrm{~h}$ (or more). The average daily ST hours was calculated using the following formula: average daily ST hours $=($ ST hours on weekdays $\times 5+$ ST hours on weekend $\times 2) / 7$. In the statistical analysis, the variable of ST was treated as continuous.

SLP was measured by the items derived from the HBSC questionnaire [20]: (1) When do you usually go to bed if you have to go to school in the next morning? (2) When do you usually go to bed at weekends or during holidays? (3) When do you usually wake up on school mornings? (4) When do you usually wake up at weekends? The answers to items 1-4 were as follows: (1) No later than 21:00;21:30;22:00; 22:30; 23:00; $23: 30 ; 24: 00 ; 00: 30 ; 01: 00 ; 01: 30 ; 02: 00$ or later; (2) no later than 21:00; 21:30; 22:00; 22:30; 23:00; 23:30; $24: 00 ; 00: 30 ; 01: 00 ; 01: 30 ; 02: 00 ; 02: 30 ; 03: 00 ; 03: 30 ; 04: 00$ or later; (3) no later than 05:00; 05:30; 06:00; $06: 30 ; 07: 00 ; 07: 30 ; 08: 00$ or later; and (4) no later than 07:00; 07:30;08:00;08:30;09:00; 09:30; 10:00; $10: 30 ; 11: 00 ; 11: 30 ; 12: 00 ; 12: 30 ; 13: 00 ; 13: 30 ; 14: 00$ or later. Participants' response was used to calculate the SLP duration of the night on weekdays and the weekend, respectively. The test retest reliability coefficient ranged from 0.58 to 0.83 . Then, the average SLP duration per night (hours) was calculated using the following formula: average SLP duration $=$ (SLP duration per night on weekdays $\times$ $5+$ SLP duration per night on weekends $\times 2$ )/7. In the statistical analysis, the variable of SLP was treated as continuous. 


\subsection{Statistical Analysis}

All the statistical analyses were conducted using SPSS version 25.0 (IBM, Corp. Armonk, NY, USA). Descriptive statistics were used to report frequency and percentage (\%) of categorical variables (e.g., sex, residence) as well as the mean and standard deviation of continuous (BMI). Pearson's chi-square test was used to examine the sex differences across all the categorical variables and student $t$-test was used to examine the sex difference across all the continuous variables. Then, Pearson correlation coefficients were calculated to determine bivariate associations among study variables. A generalized linear model (GLMs) was used to estimate the association between MVPA, ST and SLP with depression symptoms. The GLMs with Maximum Likelihood Estimation (MLE) and Robust Estimator to examine the associations between MVPA, ST and SLP with depression symptoms. To explore the association between MVPA, ST and SLP with depression symptoms reliably, three models were established (Model 1: only MVPA, ST and SLP as the independent variable; Model 2: including independent variables of Model 1 and sex, BMI, grade, ethnicity, residence as well as siblings) Model 3: all the variables of this study were included). Adjusted beta estimates were described. Statistical significance was defined as $p<0.05$ (two-sided).

\section{Results}

Tab. 1 shows the samples' characteristics of this study. The final analyses included 1331 participants $\left(51.31 \%\right.$ boys) with a mean of BMI of $18.99 \pm 4.08 \mathrm{~kg} / \mathrm{m}^{2}$. Almost all participants' ethnicity is Han (96.69\%), and most of them lived in rural areas $(72.43 \%)$ with one or more siblings $(84.3 \%)$. The average score of perceived family affluence was $3.39 \pm 0.71$. Approximately half of the parents only have received middle school education (fathers: $46.88 \%$, mothers: $48.16 \%$ ). Average MVPA, ST and SLP time of participants were $5.05 \pm 2.08$ days/week, $8.60 \pm 2.86$ hours/day, and $8.42 \pm 1.29$ hours/day, respectively. Boys were more physically active than girls $(5.43 \pm 2.16>4.65 \pm 1.92$ days/week, $p<$ $0.001)$, while girls were less sedentary than boys $(8.31 \pm 2.85<8.88 \pm 2.84$ hours/day, $p<0.001)$. The average score of depression symptoms was $37.03 \pm 5.51$, and girls reported higher average score compared to boys $(37.5 \pm 5.60>36.58 \pm 5.38, p<0.05)$.

Table 1: Sample characteristics of this study

\begin{tabular}{|c|c|c|c|c|c|c|c|c|}
\hline & & \multicolumn{2}{|c|}{ Total } & \multicolumn{2}{|c|}{ Boy } & \multicolumn{2}{|c|}{ Girl } & \multirow[t]{2}{*}{$p$} \\
\hline & & $\mathrm{n}$ & $\%$ & $\mathrm{n}$ & $\%$ & $\mathrm{n}$ & $\%$ & \\
\hline Total & & 1331 & 100 & 683 & 51.31 & 648 & 48.69 & 1 \\
\hline \multicolumn{9}{|l|}{ Sex } \\
\hline & Boy & 683 & 51.31 & I & & I & & I \\
\hline & Girl & 648 & 48.69 & & & & & \\
\hline BMI & & 18.99 & 4.08 & 19.89 & 4.53 & 18.04 & 3.30 & 0.000 \\
\hline \multicolumn{9}{|l|}{ Grade } \\
\hline & 7 & 698 & 52.44 & 352 & 51.54 & 346 & 53.40 & 0.620 \\
\hline & 8 & 309 & 23.22 & 166 & 24.30 & 143 & 22.07 & \\
\hline & 9 & 324 & 24.34 & 165 & 24.16 & 159 & 24.54 & \\
\hline \multicolumn{9}{|c|}{ Ethnicity } \\
\hline & Han & 1287 & 96.69 & 660 & 96.63 & 627 & 96.76 & 0.897 \\
\hline & Minority & 44 & 3.31 & 23 & 3.37 & 21 & 3.24 & \\
\hline \multicolumn{9}{|c|}{ Residence } \\
\hline & Urban & 367 & 27.57 & 205 & 30.01 & 162 & 25.00 & 0.041 \\
\hline & Rural & 964 & 72.43 & 478 & 69.99 & 486 & 75.00 & \\
\hline
\end{tabular}


Table 1 (continued).

\begin{tabular}{|c|c|c|c|c|c|c|c|c|}
\hline & & \multicolumn{2}{|c|}{ Total } & \multicolumn{2}{|c|}{ Boy } & \multicolumn{2}{|c|}{ Girl } & \multirow[t]{2}{*}{$p$} \\
\hline & & $\mathrm{n}$ & $\%$ & $\mathrm{n}$ & $\%$ & $\mathrm{n}$ & $\%$ & \\
\hline \multicolumn{9}{|l|}{ Siblings } \\
\hline & 0 & 209 & 15.70 & 149 & 21.82 & 60 & 9.26 & 0.000 \\
\hline & 1 & 615 & 46.21 & 306 & 44.80 & 309 & 47.69 & \\
\hline & 2 & 383 & 28.78 & 183 & 26.79 & 200 & 30.86 & \\
\hline & 3 & 94 & 7.06 & 32 & 4.69 & 62 & 9.57 & \\
\hline & 4 or more & 30 & 2.25 & 13 & 1.90 & 17 & 2.62 & \\
\hline Perceived Family Affluence & & 3.39 & 0.71 & 3.39 & 0.74 & 3.38 & 0.68 & 0.657 \\
\hline \multicolumn{9}{|l|}{ Father Educational Level } \\
\hline & Below primary school & 16 & 1.20 & 11 & 1.61 & 5 & 0.77 & 0.541 \\
\hline & Primary school & 130 & 9.77 & 62 & 9.08 & 68 & 10.49 & \\
\hline & Middle school & 624 & 46.88 & 332 & 48.61 & 292 & 45.06 & \\
\hline & High or occupation school & 423 & 31.78 & 214 & 31.33 & 209 & 32.25 & \\
\hline & Three-college & 92 & 6.91 & 42 & 6.15 & 50 & 7.72 & \\
\hline & Undergraduate & 44 & 3.30 & 21 & 3.07 & 23 & 3.55 & \\
\hline & Postgraduate or above & 2 & 0.15 & 1 & 0.15 & 1 & 0.15 & \\
\hline \multicolumn{9}{|l|}{ Mother Educational Level } \\
\hline & Below primary school & 35 & 2.63 & 21 & 3.07 & 14 & 2.16 & 0.529 \\
\hline & Primary school & 210 & 15.78 & 105 & 15.37 & 105 & 16.20 & \\
\hline & Middle school & 641 & 48.16 & 340 & 49.78 & 301 & 46.45 & \\
\hline & High or occupation school & 306 & 22.99 & 153 & 22.40 & 153 & 23.61 & \\
\hline & Three-college & 99 & 7.44 & 45 & 6.59 & 54 & 8.33 & \\
\hline & Undergraduate & 36 & 2.70 & 16 & 2.34 & 20 & 3.09 & \\
\hline & Postgraduate or above & 4 & 0.30 & 3 & 0.44 & 1 & 0.15 & \\
\hline MVPA (days/week) & & 5.05 & 2.08 & 5.43 & 2.16 & 4.65 & 1.92 & 0.000 \\
\hline ST (hours/day) & & 8.60 & 2.86 & 8.88 & 2.84 & 8.31 & 2.85 & 0.000 \\
\hline SLP (hours/day) & & 8.42 & 1.29 & 8.48 & 1.19 & 8.36 & 1.38 & 0.076 \\
\hline Depression & & 37.03 & 5.51 & 36.58 & 5.38 & 37.51 & 5.60 & 0.002 \\
\hline
\end{tabular}

MVPA: Moderate to Vigorous Physical Activity; ST: Screen Time; SLP: Sleep.

The results of correlational matrix among the study variables are presented in Tab. 2. Pearson bivariate correlations between study variables were displayed in Tab. 3. Specifically, negative correlations between MVPA and sex $(r=-0.19, p<0.01)$, perceived family affluence $(r=-0.12, p<0.01)$, mother educational level $(r=-0.08, p<0.01)$ were found in this study. Screen time was negatively correlated with sex $(r=-10, p<0.01)$ and father educational level $(r=-0.08, p<0.01)$. Sleep was positively correlated with residence $(r=0.08, p<0.01)$, and negatively correlated with father $(r=-0.08, p<0.01)$ and mother educational level $(r=-0.06, p<0.01)$. Depression symptoms was positively correlated with $\operatorname{sex}(r=0.08, p<0.01)$, and negatively correlated with residence $(r=-0.08, p<0.05)$, father $(r=-0.08$, $p<0.05)$ and mother educational level $(r=0.10, p<0.05)$. Moreover, only screen time was significantly and positively correlated with depression symptoms $(r=0.08, p<0.05)$, which revealed that more screen time was associated with the prevalence of depression symptoms. However, MVPA and sleep was not significantly correlated with the prevalence of depression symptoms. 
Table 2: Bivariate correlation matrix between study variables

\begin{tabular}{|c|c|c|c|c|c|c|c|c|c|c|c|c|c|}
\hline & 1 & 2 & 3 & 4 & 5 & 6 & 7 & 8 & 9 & 10 & 11 & 12 & 13 \\
\hline $1 \mathrm{Sex}$ & 1 & & & & & & & & & & & & \\
\hline $2 \mathrm{BMI}$ & $-0.23 * *$ & 1 & & & & & & & & & & & \\
\hline 3 Grade & -0.01 & -0.05 & 1 & & & & & & & & & & \\
\hline 4 Ethnicity & -0.00 & 0.00 & 0.02 & 1 & & & & & & & & & \\
\hline 5 Residence & $0.06^{*}$ & $-0.16^{* *}$ & $-0.08 * *$ & $-0.09 * *$ & 1 & & & & & & & & \\
\hline 6 Siblings & $0.16^{* *}$ & $-0.11 * *$ & 0.02 & 0.01 & -0.02 & 1 & & & & & & & \\
\hline $\begin{array}{l}7 \text { Perceived family } \\
\text { affluence }\end{array}$ & -0.01 & -0.05 & $0.08 * *$ & 0.00 & $0.12 * *$ & 0.05 & 1 & & & & & & \\
\hline $\begin{array}{l}8 \text { Father educational } \\
\text { level }\end{array}$ & 0.03 & -0.02 & 0.04 & -0.01 & $-0.20 * *$ & $-0.09 * *$ & $-0.13 * *$ & 1 & & & & & \\
\hline $\begin{array}{l}9 \text { Mother educational } \\
\text { level }\end{array}$ & 0.03 & $-0.05^{*}$ & 0.01 & -0.04 & $-0.14 * *$ & $-0.14 * *$ & $-0.12 * *$ & $0.49^{* *}$ & 1 & & & & \\
\hline 10 MVPA & $-0.19 * *$ & 0.05 & -0.03 & -0.05 & -0.03 & -0.04 & $-0.12 * *$ & 0.04 & $0.08 * *$ & 1 & & & \\
\hline $11 \mathrm{ST}$ & $-0.10 * *$ & 0.03 & -0.04 & 0.00 & -0.01 & -0.01 & -0.00 & $-0.08 * *$ & -0.04 & 0.01 & 1 & & \\
\hline 12 SLP & -0.05 & -0.03 & -0.00 & -0.05 & $0.08 * *$ & -0.02 & -0.04 & $-0.08 * *$ & $-0.06^{*}$ & $0.05^{*}$ & $0.09 * *$ & 1 & \\
\hline 13 Depression & $0.08 * *$ & 0.01 & -0.04 & -0.00 & $-0.08 * *$ & -0.03 & 0.01 & $-0.06^{*}$ & $-0.10^{* *}$ & 0.01 & $0.08 * *$ & -0.05 & 1 \\
\hline
\end{tabular}

MVPA: Moderate to Vigorous Physical Activity; ST: Screen Time; SLP: Sleep; ${ }^{*} p<0.05 .{ }^{* *} p<0.01$.

Table 3: The association between physical activity, screen time and sleep with depression

\begin{tabular}{|c|c|c|c|c|c|c|c|c|c|}
\hline \multirow[b]{2}{*}{ Variables } & \multicolumn{3}{|c|}{ Model 1} & \multicolumn{3}{|c|}{ Model 2} & \multicolumn{3}{|c|}{ Model 3} \\
\hline & Beta & Std. Error & $p$ & Beta & Std. Error & $p$ & Beta & Std. Error & $p$ \\
\hline Intercept & 37.66 & 1.27 & 0.000 & 36.79 & 1.95 & 0.000 & 31.55 & 2.62 & 0.000 \\
\hline MVPA & 0.03 & 0.08 & 0.689 & 0.07 & 0.08 & 0.362 & 0.11 & 0.08 & 0.167 \\
\hline ST & 0.16 & 0.06 & 0.010 & 0.18 & 0.06 & 0.004 & 0.17 & 0.06 & 0.005 \\
\hline Sleep & -0.26 & 0.14 & 0.059 & -0.20 & 0.13 & 0.128 & -0.25 & 0.13 & 0.067 \\
\hline Sex $($ ref $=$ girl $)$ & & & & -1.19 & 0.31 & 0.000 & -1.24 & 0.30 & 0.000 \\
\hline BMI & & & & 0.01 & 0.04 & 0.785 & -0.01 & 0.04 & 0.848 \\
\hline Grade $7($ ref $=9)$ & & & & 0.50 & 0.36 & 0.165 & 0.51 & 0.36 & 0.158 \\
\hline Grade $8($ ref $=9)$ & & & & 0.02 & 0.45 & 0.964 & -0.17 & 0.44 & 0.702 \\
\hline Ethnicity (ref $=$ minority) & & & & 0.36 & 0.80 & 0.652 & 0.68 & 0.85 & 0.420 \\
\hline Residence $($ ref $=$ rural $)$ & & & & 1.08 & 0.35 & 0.002 & 1.38 & 0.36 & 0.000 \\
\hline Siblings 0 (ref $=4$ or more) & & & & -0.26 & 1.18 & 0.823 & 0.56 & 1.09 & 0.608 \\
\hline Siblings 1 (ref $=4$ or more) & & & & -0.13 & 1.15 & 0.909 & 0.63 & 1.05 & 0.553 \\
\hline Siblings 2 (ref $=4$ or more) & & & & -0.81 & 1.16 & 0.483 & -0.15 & 1.06 & 0.886 \\
\hline Siblings 3 (ref $=4$ or more) & & & & -1.24 & 1.22 & 0.307 & -0.80 & 1.12 & 0.477 \\
\hline Perceived family affluence & & & & & & & 0.18 & 0.21 & 0.383 \\
\hline Father educational level $1(\mathrm{ref}=$ postgraduate or above $)$ & & & & & & & 3.54 & 2.56 & 0.167 \\
\hline Father educational level $2(\mathrm{ref}=$ postgraduate or above $)$ & & & & & & & -0.12 & 1.75 & 0.946 \\
\hline Father educational level $3(\mathrm{ref}=$ postgraduate or above $)$ & & & & & & & -0.60 & 1.67 & 0.719 \\
\hline Father educational level $4(\mathrm{ref}=$ postgraduate or above $)$ & & & & & & & -0.90 & 1.65 & 0.584 \\
\hline Father educational level $5(\mathrm{ref}=$ postgraduate or above $)$ & & & & & & & -0.80 & 1.71 & 0.638 \\
\hline Father educational level $6(\mathrm{ref}=$ postgraduate or above $)$ & & & & & & & -0.26 & 1.70 & 0.877 \\
\hline Mother educational level $1(\mathrm{ref}=$ postgraduate or above $)$ & & & & & & & 5.09 & 1.52 & 0.001 \\
\hline Mother educational level $2(\mathrm{ref}=$ postgraduate or above $)$ & & & & & & & 6.53 & 1.27 & 0.000 \\
\hline
\end{tabular}


Table 3 (continued).

\begin{tabular}{|c|c|c|c|c|c|c|c|c|c|}
\hline \multirow[b]{2}{*}{ Variables } & \multicolumn{3}{|c|}{ Model 1} & \multicolumn{3}{|c|}{ Model 2} & \multicolumn{3}{|c|}{ Model 3} \\
\hline & Beta & Std. Error & $p$ & Beta & Std. Error & $p$ & Beta & Std. Error & $p$ \\
\hline Mother educational level $3(\mathrm{ref}=$ postgraduate or above $)$ & & & & & & & 4.49 & 1.19 & 0.000 \\
\hline Mother educational level 4 ( $\mathrm{ref}=$ postgraduate or above) & & & & & & & 4.58 & 1.22 & 0.000 \\
\hline Mother educational level $5(\mathrm{ref}=$ postgraduate or above $)$ & & & & & & & 4.04 & 1.30 & 0.002 \\
\hline Mother educational level $6(\mathrm{ref}=$ postgraduate or above $)$ & & & & & & & 3.48 & 1.37 & 0.011 \\
\hline
\end{tabular}

MVPA: Moderate to Vigorous Physical Activity; ST: Scree Time; BMI: Body Mass Index; Bold Font Denotes Statistical Significance.

The results from generalized linear models for the association between MVPA, ST and SLP with depression symptoms are shown in Tab. 3. Three statistical models were used to examine the associations. In Model 1, only MVPA, ST and SLP were examined, which found ST was positively associated with depression symptoms $(\beta=0.16, p<0.05)$. MVPA and SLP were not significantly associated with depression symptoms (both $p>0.05$ ). In Model 2, variables of individual level, including sex, BMI, ethnicity, grade and number of siblings were added into the model. Consistent with the Model 1, ST was significantly associated with depression symptoms $(\beta=0.18, p<0.005)$. Sex and residence were associated with depression symptoms (both $p<0.005$ ). When parent-related variables, including family affluence and parents' educational level, were included in the Model 3, ST remained statistically significant in relation to depression symptoms $(\beta=0.17, p<0.01)$. In this model, sex and residence, as well as mother's educational level, were associated with depression symptoms.

\section{Discussion}

This study focused on the association between MVPA, ST and SLP with depression symptoms in adolescents during summertime. The current study mainly found that ST was an important correlate of depression symptoms in adolescents during summertime; but, MVPA and SLP were not associated with depression symptoms. Our study is one of very few to assess the association between behavioral factors and depression symptoms, which could provide practical implications for depression symptoms preventions in adolescents.

Much evidence has shown that excessive ST is positively associated with depression symptoms in adolescents. For example, a recently published meta-analysis including twelve cross-sectional studies and four longitudinal studies (including 1 cohort study) revealed that there was a non-linear dose-response association between ST and depression symptoms, indicating that higher ST in adolescents was significantly associated with a higher risk of depression symptoms ( $\mathrm{OR}=1.12 ; 95 \%$ CI 1.03 to 1.22 ) [25]. In Canadian youth, duration of screen time was associated with severity of depression symptoms ( $\beta=0.23, p<0.001)$ [13]. A follow-up study also found that ST would lead to higher risks for depression symptoms in adolescents [26]. These research findings based on previously published papers can support the current study. In our study, ST was the only factor positively associated with depression symptoms when adding MVPA and SLP into the statistical model. Some possible explanations can be used for the associations between increased ST and depression symptoms in adolescents. Researchers have recognized that it is likely that adolescents who spend large amounts of time engaging in ST were socially isolating [27,28]. Moreover, it has been acknowledged that a healthy attachment orientation is crucial to healthy development [29], and increased ST can harm attachment relationships, which can negatively impact affect [30]. Another mechanism could be relating to the nature of the content, situation, or messaging of the ST exposure. For example, computer/internet use may expose adolescents to cyberbullying, and it has been shown that depression symptoms are correlates of cyberbullying among adolescents [31]. Also, as posited by social comparison theory and objectification theory [32], exposure to unattainable images that objectify the human body may contribute to feelings of depression symptoms 
$[31,33]$. Also, adolescents who spend more time in front of electronic screens have more sleeping problems [34,35], which could compromise their ability to cope with stress, resulting in increased feelings of depression symptoms or anxiety. Finally, ST can also displace time spent engaging in physical activity, which is concerning given previous findings that physical activity is associated with decreased depression symptoms [36,37]. This research finding indicates that limiting ST would be beneficial to depression symptoms prevention in adolescents, which implies that using multiple strategies to reduce ST may be effective in promoting mental health. Future studies should, especially trials studies, determine the effects of reduced ST on depression symptoms and mental health.

Interestingly, the current study found that MVPA and SLP were not significant correlates of depression symptoms in adolescents. Inconsistent with previous a large number of studies [38-42], our study indicates that MVPA is not a correlate of depression symptoms in adolescents. Specifically, a study based on Nigerian adolescents demonstrated that MVPA was linked with lower risks for depression symptoms [43]. Another study consisting of Canadian youth (nationally representative sample, $\mathrm{n}=9702$ ) suggested that being physically active (having sufficient MVPA) [44] was a negative correlate of depression symptoms. There is a large body of evidence demonstrating the positive roles of physical activity. However, our study cannot concur the previous literature. Some possible explanations could be proposed. First, some studies found that vigorous PA was positively or not associated with depression symptoms [45] while moderate PA had negatively associated depression symptoms [41]. MVPA consisted of moderate and vigorous physical, which may produce an offset effect. This would be plausible that MVPA was not associated with depression symptoms. Second, in our study, the average of days for MVPA in adolescents was about 5 days, which may not be sufficient to reach the minimum dose to fight against depression symptoms. Some studies have indicated that meeting the MVPA recommendations (having $60 \mathrm{~min}$ of MVPA per day) could help reduce depression symptoms [46]. Owing to the evidence, not having MVPA per day may not be associated with lower risks for depression symptoms. A meta-analytical study has provided evidence that sleep is an essential factor associated with depression symptoms in adolescents. Some other empirical studies also support the meta-analysis [15,47-50]. For example, a longitudinal study in Canadian youth suggested that shorter SLP was a greatly high-risk factor for depression symptoms in adolescents, especially in girls [51]. It seems that little evidence can support our research finding that SLP cannot associated with depression symptoms. Possible reasons account for this discrepancy may be owing to differences in sample characteristics, measures of depression symptoms and survey time. It is recommended to use more comparable data to determine the association between SLP and depression symptoms in adolescents, which is beneficial in synthesizing more reliable evidence.

Although this study is one of few to assess the association between movement behaviors (e.g., PA, ST and SLP) and depression symptoms in adolescents, this study should be explained in light of some inherent limitations. The first limitation is self-reported measures, which may result in recall bias and measurement error. Besides, owing to the nature of the cross-sectional study, the conclusion with causality for the association between MVPA, ST and SLP with depression symptoms cannot be a draw. Moreover, movement behavior includes light PA. Owing to measurement limitation, in the current study, we cannot measure light PA; so, in the future, more studies are encouraged to include light PA into the study analysis. The last study limitation is that the samples were mainly from Jiangsu Province, which may limit the generalizability of the research findings. Future studies are encouraged to address these limitations for robust evidence that could be used in clinical relevance.

Our study has some practical implications. The biggest one is that for depression prevention, controlling or limiting ST is may be feasible approach. However, although our study did not find the significant association of MVPA and sleep with depressive symptoms in adolescents, it is not denying the positive roles of them in preventing depression. Future studies are encouraged to replicate or negate our research findings by using more improved study design. 


\section{Conclusion}

Our study is one of the first to assess the association between movement behaviors consisting of physical activity, screen time and sleep with depression symptoms in adolescents. To conclude, the present study suggests that excessive screen time, instead of moderate to vigorous physical activity and sleep, may be detrimental to depression symptoms in adolescents. From the perspective based on the current study, it would be beneficial to reduce or limit screen time for adolescents' depression symptoms prevention and mental health promotion. Future studies should use more improved study designs to replicate or negate the negative roles of screen time in developing depression symptoms in adolescents.

Acknowledgement: The authors appreciated the study participants of this survey.

Funding Statement: The author(s) received no specific funding for this study.

Conflicts of Interest: The authors declare that they have no conflicts of interest to report regarding the present study.

\section{References}

1. Patton, G. C., Coffey, C., Sawyer, S. M., Viner, R. M., Haller, D. M. et al. (2009). Global patterns of mortality in young people: A systematic analysis of population health data. Lancet, 374(9693), 881-892. DOI 10.1016/S01406736(09)60741-8.

2. Keenan-Miller, D., Hammen, C. L., Brennan, P. A. (2007). Health outcomes related to early adolescent depression. Journal of Adolescent Health, 41(3), 256-262. DOI 10.1016/j.jadohealth.2007.03.015.

3. Thapar, A., Collishaw, S., Pine, D. S., Thapar, A. K. (2012). Depression in adolescence. Lancet, 379(9820), 10561067. DOI 10.1016/S0140-6736(11)60871-4.

4. Jane Costello, E., Erkanli, A., Angold, A. (2006). Is there an epidemic of child or adolescent depression? Journal of Child Psychology and Psychiatry, 47(12), 1263-1271.

5. Mathers, C. D., Loncar, D. (2006). Projections of global mortality and burden of disease from 2002 to 2030. PLoS Medicine, 3(11), e442. DOI 10.1371/journal.pmed.0030442.

6. Li, J. Y., Li, J., Liang, J. H., Qian, S., Jia, R. X. et al. (2019). Depressive symptoms among children and adolescents in China: A systematic review and meta-analysis. Medical Science Monitor, 25, 7459-7470. DOI 10.12659/ MSM.916774.

7. Fleitlich-Bilyk, B., Goodman, R. (2004). Prevalence of child and adolescent psychiatric disorders in southeast Brazil. Journal of the American Academy of Child \& Adolescent Psychiatry, 43(6), 727-734. DOI 10.1097/01. chi.0000120021.14101.ca.

8. Chaput, J. P., Carson, V., Gray, C. E., Tremblay, M. S. (2014). Importance of all movement behaviors in a 24 hour period for overall health. International Journal of Environmental Research and Public Health, 11(12), 1257512581. DOI 10.3390/ijerph111212575.

9. Mammen, G., Faulkner, G. (2013). Physical activity and the prevention of depression: A systematic review of prospective studies. American Journal of Preventive Medicine, 45(5), 649-657. DOI 10.1016/j. amepre.2013.08.001.

10. Raudsepp, L., Neissaar, I. (2012). Brief report: Relationships between physical activity and depressive symptoms in adolescent girls. Journal of Adolescence, 35(5), 1399-1402. DOI 10.1016/j.adolescence.2012.04.003.

11. Sund, A. M., Larsson, B., Wichstrøm, L. (2011). Role of physical and sedentary activities in the development of depressive symptoms in early adolescence. Social Psychiatry and Psychiatric Epidemiology, 46(5), 431-441. DOI 10.1007/s00127-010-0208-0.

12. Jerstad, S. J., Boutelle, K. N., Ness, K. K., Stice, E. (2010). Prospective reciprocal relations between physical activity and depression in female adolescents. Journal of Consulting and Clinical Psychology, 78(2), 268-272. DOI 10.1037/a0018793. 
13. Maras, D., Flament, M. F., Murray, M., Buchholz, A. Henderson, K. A. et al. (2015). Screen time is associated with depression and anxiety in Canadian youth. Preventive Medicine, 73, 133-138. DOI 10.1016/j.ypmed.2015.01.029.

14. Hamann, C., Rusterholz, T., Studer, M., Kaess, M., Tarokh, L. (2019). Association between depressive symptoms and sleep neurophysiology in early adolescence. Journal of Child Psychology and Psychiatry, 60(12), 1334-1342. DOI 10.1111/jcpp.13088.

15. Slykerman, R. F., Thompson, J. M. D., Coomarasamy, C., Wall, C. R., Waldie, K. E. et al. (2020). Early adolescent physical activity, sleep and symptoms of depression at 16 years of age. Acta Paediatrica, 109(7), 1394-1399. DOI 10.1111/apa.15140.

16. Raniti, M. B., Allen, N. B., Schwartz, O., Waloszek, J. M., Byrne, M. L. et al. (2017). Sleep duration and sleep quality: Associations with depressive symptoms across adolescence. Behavioral Sleep Medicine, 15(3), 198215. DOI 10.1080/15402002.2015.1120198.

17. Pedisic, Z., Dumuid, D., Olds, T. S. (2017). Integrating sleep, sedentary behaviour, and physical activity research in the emerging field of time-use epidemiology: Definitions, concepts, statistical methods, theoretical framework, and future directions. Kinesiology, 49(2), 252-269. DOI 10.26582/k.49.2.14.

18. da Costa, B. G. G., Chaput, J. P., Lopes, M. V. V., Malheiros, L. E. A., Silva, K. S. (2020). Movement behaviors and their association with depressive symptoms in Brazilian adolescents: A cross-sectional study. Journal of Sport and Health Science. DOI 10.1016/j.jshs.2020.08.003.

19. Lee, E. Y., Spence, J. C., Tremblay, M. S., Carson, V. (2018). Meeting 24-hour movement guidelines for children and youth and associations with psychological well-being among South Korean adolescents. Mental Health and Physical Activity, 14, 66-73. DOI 10.1016/j.mhpa.2018.02.001.

20. Liu, Y., Wang, M., Tynjälä, J., Lv, Y. Villberg, J. et al. (2010). Test-retest reliability of selected items of health behaviour in school-aged children (HBSC) survey questionnaire in Beijing, China. BMC Medical Research Methodology, 10(1), 73. DOI 10.1186/1471-2288-10-73.

21. Chen, S. T., Liu, Y., Hong, J. T., Tang, Y., Cao, Z. B. et al. (2018). Co-existence of physical activity and sedentary behavior among children and adolescents in Shanghai, China: Do gender and age matter? BMC Public Health, 18 (1), 1287. DOI 10.1186/s12889-018-6167-1.

22. Shen, H., Yan, J., Hong, J. T., Clark, C., Yang, X. N. et al. (2020). Prevalence of physical activity and sedentary behavior among Chinese children and adolescents: Variations, gaps, and recommendations. International Journal of Environmental Research and Public Health, 17(9), 3066. DOI 10.3390/ijerph17093066.

23. Chen, S. T., Yan, J. (2020). Prevalence and selected sociodemographic of movement behaviors in schoolchildren from low-and middle-income families in Nanjing, China: A cross-sectional questionnaire survey. Children, 7(2), 13. DOI 10.3390/children7020013.

24. Chen, S. T., Liu, Y., Tremblay, M. S., Hong, J. T., Tang, Y. et al. (2020). Meeting 24-hour movement guidelines: Prevalence, correlates and the relationships with overweight and obesity among Chinese children and adolescents. Journal of Sport and Health Science, 2(7S), 581. DOI 10.1016/j.jshs.2020.07.002.

25. Liu, M., Wu, L., Yao, S. (2016). Dose-response association of screen time-based sedentary behaviour in children and adolescents and depression: A meta-analysis of observational studies. British Journal of Sports Medicine, 50(20), 1252-1258. DOI 10.1136/bjsports-2015-095084.

26. Gunnell, K. E., Flament, M. F., Maras, D., Buchholz, A., Henderson, K. A. et al. (2015). Associations between screen time, physical activity, and depression in adolescents: A four-year longitudinal real study. Journal of Sport \& Exercise Psychology, 37(2), S117. DOI 10.1123/jsep.2014-0093.

27. McHale, S., Crouter, A. C., Tucker, C. (2000). Free-time activities in middle childhood: Links with adjustment in early adolescence. Child Development, 72(6), 1764-1778. DOI 10.1111/1467-8624.00377.

28. Bohnert, A., Garber, J. (2008). Prospective relations between organized activity participation and psychopathology during adolescence. Journal of Abnormal Child Psychology, 35(6), 1021-1033. DOI 10.1007/s10802-007-9152-1.

29. Scharfe, E., Eldredge, D. (2016). Associations between attachment representations and health behaviors in late adolescence. Journal of Health Psychology, 6(3), 295-307. DOI 10.1177/135910530100600303. 
30. Richards, R., McGee, R., Williams, S., Welch, D., Hancox, R. (2010). Adolescent screen time and attachment to parents and peers. Archives of Pediatrics \& Adolescent Medicine, 164(3), 258-262. DOI 10.1001/ archpediatrics.2009.280.

31. Kowalski, R., Limber, S. (2013). Psychological, physical, and academic correlates of cyberbullying and traditional bullying. Journal of Adolescent Health: Official Publication of the Society for Adolescent Medicine, 53(1), S13S20. DOI 10.1016/j.jadohealth.2012.09.018.

32. Fredrickson, B., Roberts, T. A. (1997). Objectification theory: Toward understanding women's lived experiences and mental health risks. Psychology of Women Quarterly, 21(2), 173-206. DOI 10.1111/j.1471-6402.1997.tb00108.x.

33. Tiggemann, M., Kuring, J. (2004). The role of body objectification in disordered eating and depressed mood. British Journal of Clinical Psychology, 43(3), 299-311. DOI 10.1348/0144665031752925.

34. van den Bulck, J. (2004). Television viewing, computer game playing, and internet use and self-reported time to bed and time out of bed in secondary-school children. Sleep, 27(1), 101-104. DOI 10.1093/sleep/27.1.101.

35. Nelson, M., Gordon-Larsen, P. (2006). Physical activity and sedentary behavior patterns are associated with selected adolescent health risk behaviors. Pediatrics, 117(4), 1281-1290. DOI 10.1542/peds.2005-1692.

36. Moor, M., Beem, A. L., Stubbe, J., Boomsma, D., Geus, E. (2006). Regular exercise, anxiety, depression and personality: A population-based study. Preventive Medicine, 42(4), 273-279. DOI 10.1016/j.ypmed.2005.12.002.

37. Ströhle, A. (2008). Physical activity, exercise, depression and anxiety disorders. Journal of Neural Transmission, $116(6), 777-784$.

38. Schuch, F. B., Vancampfort, D., Firth, J., Rosenbaum, S., Ward, P. B. et al. (2018). Physical activity and incident depression: A meta-analysis of prospective cohort studies. American Journal of Psychiatry, 175(7), 631-648. DOI 10.1176/appi.ajp.2018.17111194.

39. Korczak, D. J., Madigan, S., Colasanto, M. (2017). Children's physical activity and depression: A meta-analysis. Pediatrics, 139(4), 2016-2266.

40. McMahon, E. M., Corcoran, P., O’Regan, G., Keeley, H., Cannon, M. et al. (2017). Physical activity in European adolescents and associations with anxiety, depression and well-being. European Child \& Adolescent Psychiatry, 26 (1), 111-122. DOI 10.1007/s00787-016-0875-9.

41. Kleppang, A. L., Hartz, I., Thurston, M., Hagquist, C. (2018). The association between physical activity and symptoms of depression in different contexts-A cross-sectional study of Norwegian adolescents. BMC Public Health, 18(1), 1368. DOI 10.1186/s12889-018-6257-0.

42. Gianfredi, V., Blandi, L., Cacitti, S., Minelli, M., Signorelli, C. et al. (2020). Depression and objectively measured physical activity: A systematic review and meta-analysis. International Journal of Environmental Research and Public Health, 17(10), 3738. DOI 10.3390/ijerph17103738.

43. Adeniyi, A. F., Okafor, N. C., Adeniyi, C. Y. (2011). Depression and physical activity in a sample of Nigerian adolescents: Levels, relationships and predictors. Child and Adolescent Psychiatry and Mental Health, 5(1), 16. DOI 10.1186/1753-2000-5-16.

44. Belair, M. A., Kohen, D. E., Kingsbury, M., Colman, I. (2018). Relationship between leisure time physical activity, sedentary behaviour and symptoms of depression and anxiety: Evidence from a population-based sample of Canadian adolescents. BMJ Open, 8(10), e021119. DOI 10.1136/bmjopen-2017-021119.

45. Allison, K. R., Adlaf, E. M., Irving, H. M., Hatch, J. L., Smith, T. F. et al. (2005). Relationship of vigorous physical activity to psychologic distress among adolescents. Journal of Adolescent Health, 37(2), 164-166. DOI 10.1016/j. jadohealth.2004.08.017.

46. Poitras, V. J., Gray, C. E., Borghese, M. M., Carson, V., Chaput, J. P. et al. (2016). Systematic review of the relationships between objectively measured physical activity and health indicators in school-aged children and youth. Applied Physiology Nutrition and Metabolism, 41(6), S197-S239. DOI 10.1139/apnm-2015-0663.

47. Ogawa, S., Kitagawa, Y., Fukushima, M., Yonehara, H., Nishida, A. et al. (2019). Interactive effect of sleep duration and physical activity on anxiety/depression in adolescents. Psychiatry Research, 273, 456-460. DOI 10.1016/j.psychres.2018.12.085.

48. Stearns, M., Wilkerson, A., Speed, K. J. (2020). Adolescent sleep mediates maternal depression and harsh parenting. Sleep, 43, A359. DOI 10.1093/sleep/zsaa056.941. 
49. Zhu, D., Dai, G., Wang, F., Geng, J., Xu, D. et al. (2015). The comparison of sleep quality and depression after Tai Chi intervention on methamphetamine-type drug abusers in China. Medicine and Science in Sports and Exercise, 47(5), 673. DOI 10.1249/01.mss.0000478559.59702.d0.

50. Conklin, A. I., Yao, C. A., Richardson, C. G. (2018). Chronic sleep deprivation and gender-specific risk of depression in adolescents: A prospective population-based study. BMC Public Health, 18(1), 724. DOI 10.1186/s12889-018-5656-6.

51. Guan, S. S. A., Bower, J. E., Almeida, D. M., Cole, S. W., Dahl, R. E. et al. (2016). Parental support buffers the association of depressive symptoms with cortisol and C-reactive protein during adolescence. Brain Behavior and Immunity, 57, 134-143. DOI 10.1016/j.bbi.2016.03.007. 